\title{
The Role of Chronemic Agency in the Processing of a Multitude of Mediated Conversation Threads
}

\author{
Yoram M Kalman \\ The Open University of Israel \\ yoramka@openu.ac.il
}

\author{
Ana M Aguilar \\ University of Texas at Austin \\ ana.m.aguilar@utexas.edu
}

\author{
Dawna I Ballard \\ University of Texas at Austin \\ dballard@austin.utexas.edu
}

\begin{abstract}
Hundreds of messages and conversations stream daily into our communication media and devices. How do we manage this influx without missing urgent messages? In this study we provide initial evidence that this is achieved by closely monitoring only a small number of media-those media where users expect time sensitive messages to arrive. We describe this heightened attention to a medium as "assigning the medium high chronemic agency", and characterize chronemic agency through extensive interviews of eighteen American undergraduate students. Our findings reveal how chronemic agency is involved in communication norms associated with urgency. Furthermore, they demonstrate the dynamic nature of chronemic agency, and its role in managing responsiveness and in dealing with communication overload. We conclude with a discussion of the shifting nature of the synchronicity of mediated conversation.
\end{abstract}

\section{Introduction}

How do we effectively manage the stream of conversations and messages that flow between our various digital devices and applications? According to several Pew surveys [1-3], American adults and teens use a large number of media, most of which are also installed on their smartphones, to send and receive messages from peers, family and others, throughout the day. For example, in a week-long survey [3], smartphone users were contacted twice a day in diverse hours, (for a total of 14 times during the full week) and asked about smartphone use in the preceding hour. On average, participants reported in seven out of the 14 that they used text messaging in the recent hour, 5.3 out of 14 that they used email, 4.2 out of 14 that they recently used social networking, and 4.1 out of 14 that they recently used voice/video calling. Young adults' (18-29) use of texting and email was even higher.

Every day hundreds of messages emerge and accumulate in our email inboxes, social media accounts, texting apps, and other forms of digital media [4, 5]. Constantly monitoring all of these channels requires a great deal of time and energy [6]. Nonetheless, in the "always on" culture, individuals are expected to be reachable and responsive throughout the day, and to be able to promptly respond to urgent messages. How is this achieved? We demonstrate that this challenge is met by users prioritizing some media over other media, and closely monitoring these media more frequently for incoming notifications and messages. We propose describing this preferential treatment of specific media as assigning them high chronemic agency. In this report we briefly describe this novel concept of chronemic agency and its theoretical foundations, and present findings of a study designed to elicit evidence for perceptions of chronemic agency in a sample of undergraduate students and for the role of chronemic agency in the social norms of mediated communication.

\subsection{Chronemic agency}

Chronemic agency is a novel concept in communication studies. Chronemics is the study of the role of time in communication [7], and agency is used in the sense of the capacity to exert power [8]. Flaherty [9] describes temporal agency as stemming from human agency - the agency of people over time, or from non-human agency - the agency of time over people. The term chronemic agency is used to denote temporal agency in communication, and we propose that the chronemic agency of a communication medium expresses the relative power that messages and notifications that are received through the medium have on the recipient's attention and reaction time. Chronemic agency expresses the attitude of a user towards a communication medium-how that user monitors incoming messages on that medium, and how s/he acts on those messages. Thus, medium A has higher chronemic agency than medium B for a specific user if this user monitors medium A more frequently than medium B and can consequently act on messages and notifications from medium A more quickly than on messages and notifications from medium B.

For example, if a specific user monitors her incoming text (SMS) messages throughout the day, so 
that she can quickly respond to urgent incoming messages, but checks her Facebook feed only several times a week, then for this user the chronemic agency of texting (medium A) is higher than that of Facebook (medium B). Chronemic agency is not a characteristic of the medium. Rather, it describes the attitude of users towards their communication media. It is relative in that there is no absolute measure of the chronemic agency of a specific medium, and it is measured in relation to other media.

In conceptualizing this work we synthesized key aspects of temporal agency and chronemics research through the theoretical lens of social entrainment. Entrainment is a concept with origins in biology that has been used by social scientists to study the ways in which human interaction is inherently temporal, or time-bound. Notably, social entrainment theory considers how endogenous pacers (one's own rhythms) are influenced by exogenous pacers (the timing and patterns of interaction with others). For instance, in the life of a college student, there are many exogenous pacers (class schedule, midterms and finals, weekend parties and visiting with friends, semester breaks, family holidays, etc.). Ultimately, entrainment occurs when an exogenous pacer exerts enough influence to lead to changes in our endogenous rhythms.

\section{Goals and hypotheses}

The essence of interactive human communication is the exchange of messages: receiving messages, and responding to them. The chronemic aspects of the response (i.e., the timing of the response) are an integral component of the message. Traditionally, communication was either synchronous (the response immediately follows the message) or asynchronous (the response is delayed). Under those circumstances, a key way to communicate the high urgency of a message was to use a synchronous medium. But now that we receive a constant stream of messages that are transmitted instantaneously, our question is how users still succeed in communicating high urgency. We hypothesize that this is achieved by a generally accepted understanding that only a subset of the media have high chronemic agency, and by using these media for urgent messages. This is accompanied by an understanding that other, lower chronemic agency media, are for less urgent messages.. If this is so, (H1) we expect users to be able to place the different media they use on a continuum of urgency, and (H2) we expect users who belong to a given social group, whose members continually communicate with each other, to exhibit communication norms (a consensus) about the relative placement of specific media on the urgency continuum.
The goals of this exploratory study were to propose an operational definition of chronemic agency and to perform in depth semi-structured exploratory interviews with members of a relatively homogenous social group in an effort to find support for $\mathrm{H} 1$ and $\mathrm{H} 2$, as well as to identify issues associated with chronemic agency for future research.

\section{Method}

Undergraduate students were recruited at a southwestern university in the United States in exchange for extra course credit. Participation was voluntary and participants could withdraw at any time. Data were collected using interviews followed by diagraming. A total of 18 semi-structured interviews were conducted that lasted between 42-60 minutes. Interviews were audio recorded and transcribed. After choosing a pseudonym followed by a number (e.g. John55), interviewees were prompted to list all the different communication media they use and were then asked a series of questions regarding their practices and usage of each individual medium. After the first five interviews, the protocol was augmented by asking participants $(n=13)$ to complete a diagram in which they would place each medium they had discussed, on a continuum between "Not Urgent At All" and "Very Urgent". According to Bagnoli [10], "The inclusion of non-linguistic dimensions in research, which rely on other expressive possibilities, may allow us to access and represent different levels of experience" (p. 547). We report on an analysis of the 18 interviews (Undergraduate participants: 19-22 years old; 15 female, 3 male; 9 White/Caucasian, 5 Hispanic/Latino, 2 Asian/Pacific Islander, 1 Black/African American, 1 other; 1 international student) resulting in 401 pages of interview transcriptions and diagrams.

The interviews were analyzed using a thematic coding process in which the coder is immersed in the data in order to identify themes that emerge in relation to the research question [11]. Two researchers conducted the coding using qualitative coding software Atlas.ti, and a third researcher read the transcripts but did not actively code them. During the process of coding, findings were routinely shared between the three researchers in order to discuss and refine codes. One round of coding was performed after responses were grouped based on media type (e.g. Facebook, Instagram, email, text, etc.). Another round of coding was based on participant's usage of time and pacingrelated words such as: immediate, soon, delay, later, urgent and their derivatives. During the process, codes were compared across media to identify similarities or differences, and themes were identified. The analysis included the transcripts and the hand-drawn diagrams. 
In addition to the thematic analysis, the 13 diagrams drawn by the participants were also quantitatively analyzed. The distance between the lowest ranked medium and each of the other media was manually measured with a millimeter ruler. The distances were converted to decimal fractions so that for each participant the lowest ranked medium was zero, and the highest ranked medium was one. A fraction in the range 0-1 represented the relative chronemic agency of that medium. For example, if a participant ranked four media, with Facebook ranked as the lowest chronemic agency, then Instagram $30 \mathrm{~mm}$ to its right, texting $85 \mathrm{~mm}$ to the right of Facebook, and phone call $100 \mathrm{~mm}$ to the right of Facebook, the chronemic agency of Facebook for this participant would be 0, of Instagram .3 , of texting .85 and of phone call 1.0. For each medium that was classified by at least five of the participants, we calculated the mean chronemic agency and the standard deviation.

\section{Results}

All participants were asked what medium would another student or friend use if they wished to get in touch with them urgently. The responses were very consistent, with the majority of participants saying that calling is the best method, a few more saying that texting (messaging) is the best, and some saying either calling or texting.

The diagrams drawn at the end of the interview (e.g. Figure 1), portray a more complex picture. Sometimes more media are mentioned in the "very urgent" side of the diagram, but phoning and texting are still the clear consensus media for urgent contact in the social group studied here. A possible explanation for the differences between the spoken responses and the diagrams is that the spoken responses were to the specific question, about a peer or friend who wishes to contact them urgently, while the diagraming was a concluding exercise that followed a discussion of many media and many social circles beyond friends and peers. The "top of mind" answer for most participants was clearly calling and/or texting. The quantitative analysis ${ }^{1}$ of the diagrams (Figure 2) reinforces the conclusion that calling and texting have the highest chronemic agency (.99 and .82, respectively) and a high consensus (low SD), while the other media have lower chronemic agency (.65 and below), and usually a higher SD.

Following these findings, a thematic analysis of the interview transcripts revealed several key themes related to chronemic agency and to its assignment to different communication media. The central theme that

\footnotetext{
${ }^{1}$ Given the small sample size, this analysis is better described as a quantitative demonstration of qualitative findings.
}

emerged was that chronemic agency of media has an important role in the communication norms that participants described, and that chronemic agency is relative and dynamic. This dynamism allows the members of this social group to effectively cope with the unrelenting flow of messages in multiple channels that is the everyday reality of American undergraduate students, as well as of knowledge workers and of others in the knowledge society. Note that our sample is neither random nor representative. Nevertheless, the participants do represent individuals coping with communication challenges experienced by many students and knowledge workers.

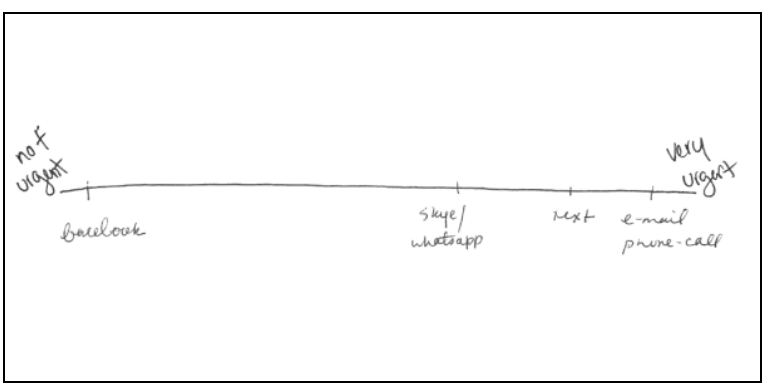

Figure 1: The diagram drawn by Traveller89. Facebook was ranked as the medium with the least chronemic agency, and then from left to right: Skype and WhatsApp (same ranking), text, and finally email and phone call (same ranking).

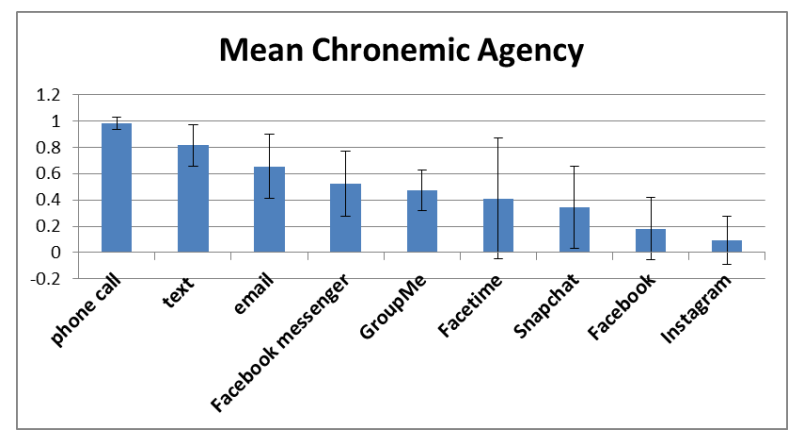

Figure 2: Mean chronemic agency of the nine most common communication media. Error bars mark +/one standard deviation (SD).

\subsection{High chronemic agency}

Participants were quick, decisive and consistent when giving the answer that calling or texting are the media that peers or friends should use to get in touch with them urgently. This understanding is implicit, and it is usually not something students feel they need to tell each other. 
Interviewer: Do you tell them to call you instead of let's say a text message? How do they know that's the best way to reach you?

Michelle1: I don't tell them. I'm pretty sure just based on how ... How can I say it? Because whenever I'm with them physically, they always see me with my phone on my hand. I mean, I'm pretty sure that they will know that if I call them ... Even they see me that a lot of people call me, and instead of texting me if they need something quickly ...

Interviewer: Do you tell them that that's the best way to reach you?

Jersey8: No. It's just kind of implied.

Interviewer: How do they know to do that?

Saral: I usually let people know, my friends they know if they ... If they're my friends they already know. I'm a mentor so I tell my students if they ever need to reach me, that they can always text me or call me so they know that mode of communication as well.

Interviewer: Do you specifically tell them that if they need to get in contact with you, to call you?

Girl96: No. My primary form of communication with my friends is via text message.

Interviewer: Out of the ones that you mentioned, text message or a phone call, is there one that they prioritize?

Girl96: Text message would just be more of like updates or just normal conversation, but if they had to get ahold of me urgently and had to get an answer from me immediately, then a phone call would be the better form of communication for that.

Interviewer: All right. How do they know that that's the best way to reach you, though?

ReginaFlangee 1: I generally tell them or they figure out after awhile. I know my boyfriend figured out after all the stuff ... He'd generally send me a text message and just to clarify things. I was just like, "I'm not going to deal with this." I just ended up calling, so finally he just realized basically just the best way to get a hold of me is to call me.

\subsection{Lower chronemic agency}

There was also some consensus about which media have lower chronemic agency: for example, it is quite clear that in this group of undergraduate students Facebook and Instagram are not used for urgent messaging, are not checked as often, and belong in the middle or "not urgent" side of the diagrams drawn by the participants.
Interviewer: Can you tell me about how you use that [Facebook] on a typical weekday?

JohnSmith8: When I'm waiting in between classes or just when I'm bored, I just read through it. I never post anything. I just use it to just see what other people are up to.

Traveller89: ...Facebook, honestly, I only got it when I transferred here [to university]. I didn't really have one before. I just got it because, just like joining different organizations and stuff, they post a lot of things on Facebook. Announcements, reminders, or anything. I got it for that purpose and mostly for school purposes. I don't really tend to check it that much. Stuff that gets sent to Facebook by my organizations also gets sent to my email, so I know from my email before my Facebook. Facebook, I check it maybe like once or twice a week.

Karina4: I actually tend to use it [Facebook] more in the evening once I'm done with classes because I've trained myself not to check Facebook as rigorously during the day because it's a time-suck for me and then I just get lost in it. I keep it for nighttime when I'm done studying and sort of need to decompress a little bit. I'll just scroll through my Facebook feed.

Interviewer: Okay. Instagram. How are you using Instagram on a typical weekday?

McKenzieEmery13: Several times a day. It's my favorite app. I will mindlessly log into it, maybe ten times a day.

...

Interviewer: How often do you check for new messages?

McKenzieEmery13: Every time I log on, so ten times a day. I don't check for new messages. I'm looking at new content. Then if I have a notification that there's a new message, I'll check it but I don't get notifications on my phone of new content or notifications from Instagram at all.

Interviewer: Is that intentional?

McKenzieEmery13: Yes.

Interviewer: Because?

McKenzieEmery 13: It's distracting. I don't like to be told every time someone liked my photo because I feel the need to log onto my phone every time but I don't need to log on to look at the photo I posted 200 times.

Interviewer: Okay. What would be something that does require a response on Instagram? On this public side, whether it's someone tagging you I guess.

McKenzieEmery13: Something, like a very direct personal message that was like ... I don't know. I can't think of anything really. A very direct personal 
message but people don't comment on Instagram because it's a public sphere...

Interviewer: What about Instagram? How do you use Instagram on a typical weekday?

Girl96: I don't check Instagram every day. I get notifications every day that I'll swipe away on my home screen, but I don't necessarily go through my Instagram feed every single day, it depends because I'm doing other things that take precedent over doing that, so usually when I'm bored or on the weekends I'll check it a lot more than I do during the weekdays because I'm not as busy on the weekends as I am during the week.

\subsection{How chronemic agency works}

In the following quotations we see hints as to how differential chronemic agency enables the undergraduate students to better manage the constant flow of a large number of messages. A very small number of media are those that will be used for urgent communications. These media are monitored on an ongoing basis throughout the day, and urgent messages/calls are identified and acted upon almost in real time.

Interviewer: If another student or a friend of yours needs to get in touch with you urgently, how do they reach you?

JohnSmith8: Probably, call. I try to answer my phone as much as possible whenever I can. If a friend or a family member needs to get a hold of me, they call me.

Interviewer: How do they know that's the best way to reach you?

JohnSmith8: Let me think about it. If it's urgent, they know that I'll try and pick up my phone whenever I can. Actually, now that I'm thinking of it, maybe it's text because a lot of my family and friends know that I'm in classes throughout the day. If they needed to get a hold of me, they'll send me something saying, "Hey, blah, blah, blah." I'll probably respond to that because it's more discreet. If I'm in class, I can respond to it or if I'm at work, I can quickly type something out. If it is something serious, then I can call. I think if it is urgent, they usually text me.

Interviewer: How often do you check for new messages?

Saral: Whenever I'm on my phone. I don't know. Whenever I glance at my phones. If it's in between classes or maybe after my class or even I still sometimes just press my home screen to see what time it is and then I can see a message there but if I'm in class I'll probably try to wait after if I knew it's something that can wait or I'll reply then. Yeah, my phone is always nearby so even if I don't have it, if it goes off I could hear it and then I'll check it.

\subsection{When to check?}

The differential chronemic agency assigned to communication media helps separate the communication streams into those that need to be monitored constantly and those that do not, but that is not to say that the lower chronemic agency media are ignored. Although they do not need to be monitored very closely, messages and notifications on these media still need to be managed effectively to stay on top of things. This is accomplished through a system developed by each student based on their circumstances and preferences. These systems are geared to integrate mediated communication into students' everyday routines in a way that will enable them to receive and send messages throughout the day without unnecessary stress. We have already seen how McKenzieEmery13 and Karina4 describe how they use Instagram and Facebook during downtime. Participants also described how they use some media during specific times of the day:

McKenzieEmery13: I watch [Snapchat] stories maybe in the morning and then in the evening so they have enough time to load, then I'll watch them all at once, look at the photos I received, respond or no, just decide if I want to, then I'll put it away.

Interviewer: You do that, you said once a day?

McKenzieEmery13: Once or twice, twice generally: in the morning and then in the afternoon.

Interviewer: Okay. That's when you check for all the direct messages?

McKenzieEmery13: Yeah.

Interviewer: Is there a reason why you do that?

McKenzieEmery13: Just to minimize the amount of time I spend on the app.

Interviewer: Do you have notifications set up for that?

McKenzieEmery13: No.

Others will use the medium several times a day. For example:

Corgi19: I check for new [email] messages everyday because one time I made the mistake of not checking my email and I got to class and class was canceled that day and I woke up really early and did a lot of scrambling to get to that class. Now, I try to check it as much as I can first thing when I wake up 
just to make sure I know what's going to be happening for the day or what's not going to be happening.

Interviewer: You check it in the morning and then are you checking it throughout the rest of the day?

Corgi19: Yeah, throughout the rest of day, sometimes maybe like once every 4 to 5 hours. Then definitely in the evening time, I scroll through and see if I miss anything.

JohnSmith8: I would say, in a day, I check my email about 20 to 30 times.

Interviewer: Is there any rhyme or reason to when you're checking it?

JohnSmith8: Checking for updates with professors or maybe a class gets cancelled. I'm checking for work. I'm seeing if there's going to be something that might be beneficial for me. My adviser might be sending me good news.

Interviewer: How are you checking for these new messages?

JohnSmith8: I'd scroll down on my phone and it will load it, mainly. If I'm waiting in between classes, I'll pull out my computer and double check that and read through anything that I missed.

Interviewer: How do you use Snapchat throughout the week?

Barbara7: I use it a lot. I probably check it every 3 hours. At least, that's just me checking like if I get an alert, I usually look at it immediately.

Interviewer: When you say you're checking it, what are you exactly doing?

Barbara7: Waiting to see if somebody opened up my message or to see if story is updated or et cetera.

Note that Barbara7 is not only checking for incoming messages but also monitoring who viewed her messages.

Another possibility is to use an application once a certain number of notifications of new messages accumulated:

Interviewer: Is there rhyme or reason to when you check it? [Twitter]

Barbara7: No, I usually wait till I have a couple alerts and then I'll be like, "Oh, okay. I should probably keep up with that." It's probably just like the amount of alerts I get that prompts me to check it.

Interviewer: Is there a certain amount of alerts?

Barbara7: Like five. Once I get five and I'll go, "Okay, you should probably get on there and take care of business."
Furthermore, the chronemic agency can vary not only between media, but also for the same medium at different times, for example when school is on, versus during vacation:

ReginaFlangee 1: ...During the summer, I don't really check it all that much. When school is in session, I feel like there's always something going on, there's something I need to know about, or there's something being added, especially since I get alerted about my grades through email also from Canvas [the university's internal information system]. I check my email in a very OCD way.

Interviewer: You're checking it during the school year mostly for ... When you're saying that there's a lot going on, what do you mean?

ReginaFlangee 1: There's a lot of events around campus. I really like to know my grades. I will sit there and check Canvas all the time. It's also nice to sit there and get text messages from Canvas and also emails to where I'll see it on the home screen. It's even then I'll sit there and I'll just check my email four or five times again an hour. Yeah, it's just, it's different during the school year. During the summer, I'll check it like twice a day probably.

Interviewer: Why?

ReginaFlangee 1: Just because there's not that much going on. Generally by that time, I'm working to where there's not as much I feel like I need to be aware of and there's not as many things going to my account that I really don't already know about. (...) Very rarely is it actually something that's very important.

\subsection{Responding quickly}

When participants describe the variety of strategies that determine when and how to access their different media, there is a consistent theme of aiming to minimize the time between opening the message and responding to it. Note that by responding we mean acting on a message - answering, or taking another action.

Interviewer: How quickly do you tend to respond to e-mails?

Michelle1: As soon as I open them.

Interviewer: How often do you check for new [voice] messages?

JohnSmith8: Whenever they pop up if I missed a call.

Interviewer: When you do receive a message, what do you typically do? 
JohnSmith8: I'll listen to it, figure out who was calling, make sure they're calling the right number, and then call them back to clear up anything.

Interviewer: How quickly do you respond?

JohnSmith8: After I've read or listened to the message. I would probably say within five to 10 minutes.

Interviewer: How often do you check for new [Snapchat] messages?

Longhorn 1: I have notifications from Snapchat so in free time, when I check my phone, I'll look at them and respond if I have time to do that but I also will let them sit and not check them until I have free time.

Flower3: Yeah. Well, once I see a [text] message, I usually respond right away because I'm on my phone anyway.

Interviewer: You respond right away just ... Is there a reason for it or ...

Flower3: No, just because- Well, I don't know. I feel like people who don't, like if you're on your phone anyway, unless you're purposefully trying to ignore someone or something, I don't know why you wouldn't answer them right away unless you are busy and doing something. But, usually, like if I have enough time to check the message, I'm going to have enough time to respond to it.

One of the key reasons given to responding immediately is that waiting with a response increases the likelihood of forgetting to respond or losing the thread. This is not surprising, in light of the large number of daily messages:

Flower3: Sometimes, if a message is really long and I read it but I am busy doing something else, I'll get distracted, do something else, and then completely forget, and then realize a few days later, "Oh my God, I completely forgot to respond to your text. Sorry."

Longhorn 1: I feel like if you don't respond as quickly, because the [Snapchat] message goes away, people forget what they send. If you respond faster then people know your response and they can remember what they said.

Interviewer: Okay. When you do need to respond to a [GroupMe] message, how quickly do you tend to respond?

Longhorn 1: Usually as soon as I see it.

Interviewer: Is there a reason for that?

Longhorn1: Sometimes GroupMe messages can get lost because there are so many people. I have one message with over thirty people and so if people are constantly responding then you have to scroll up. You lose track of the conversation.

Interviewer: When you're deciding that you are going to respond to these calls, how quickly do you tend to respond?

McKenzieEmery 13: Immediately or I forget.

Interviewer: Immediately or you forget, okay. Can you tell me of a time when you should have answered or returned a phone call more quickly but did not?

McKenzieEmery13: Yeah. A couple of weeks ago, my grandfather called me twice and I forgot to call him back. Then he surprised, he was coming to [the city where the campus is based]. He was in [the city], I didn't know. I still saw him but I had plans that I had to cancel because I had to go see my grandfather.

Interviewer: Why did you not answer or return the call quickly?

McKenzieEmery13: I was busy and then forgot to call him back.

Another important reason for not delaying a response has to do with managing the sender's impressions of the recipient, who in many cases wishes to present him or herself as highly responsive. Often, this wish to respond quickly is mentioned in the context of the "read notification"-Tools that allow the sender to see whether (and/or when) the recipient has opened the message.

Interviewer: How quickly do you respond to a [Facebook] message if it requires a response?

Jersey8: Usually immediately because they can see that I read it, and I feel uncomfortable not answering right away.

Interviewer: Why?

Jersey8: I feel like some people take that the wrong way, even though it's not meant to be, "Oh, I read it. I'm ignoring you." If I did read it ... I don't know. Sometimes I interpret it wrong, but I could just be busy or they could just be busy. I try to do it quickly so that there's no room for that.

Barbara7: I was to take up an interview for one of these internships and I did it a couple times and I would get back to people within 2 minutes or 3 minutes just like, "Oh, this is my availability". "This is what I could do," and just setting up where to park and what the building is and stuff like that. It's really like we're getting back and forth to each other pretty quickly and so it made for me looking like I actually look at my email as opposed to some people who I guess take forever to respond. It's hard to get information across. 
Interviewer: What was your reason for responding so quickly?

Barbara7: I just wanted to get the information across quickly so I looked like I was put together or something.

Interviewer: Okay, what do you typically do when you have a new [text] message?

Saral: I open it and I read it and then I just respond to it accordingly. If they're asking me something, I respond to their question, if they just want to talk, I'll just talk so it just depends on what the message is asking of me.

Interviewer: How quickly do you respond?

Saral: I respond very quickly, if I see a message from someone I'll respond to them if I can. If I have my phone and I'm not doing anything, I can respond to you then. It's not going to be too long if someone texts me unless it's something that ... I know sometimes my co-workers will ask if we can switch shifts or anything and then if it's something I have to go and check my calendar, I'll text them back I got their message so I'll text them back let me look at my schedule and I'll get back to you and then ... I just let them know that I received your message and then I get back to them later on where I can.

One participant demonstrated the importance of managing these impressions when she described an elaborate process she goes through to open and read a Snapchat message without the sender's knowledge:

Macarena5 ...you have to turn airplane mode, open the Snapchat, delete the application, turn back on the internet, install the application and $\log$ in again. It's a whole process that really makes you think like, "Is it really worth it going through the whole process just to avoid that person knowing that you saw a Snapchat at the time that you did?"

Interviewer: That's interesting. This is just so that they don't know when you opened it?

Macarena5 : It's just so that they don't know that you opened it or if you want to see it right away but you need to give it a couple of hours not to show that much interest so you would go through that process.

\section{Discussion and conclusion}

This study explored the role of chronemic agency in the daily communication activities of members of a relatively homogenous social group. The interviews provide extensive support for our hypothesis $(\mathrm{H} 1)$ that users are able to place the different media they use on a continuum of urgency. The interviews provide partial support for our hypothesis $(\mathrm{H} 2)$ that the participants exhibit communication norms (a consensus) about the relative placement of specific media on the urgency continuum. This consensus was clearly evident in the context of high chronemic agency media, particularly texting and calling. There was a weaker consensus in regards to other, lower chronemic agency media, with the exception of GroupMe, where there was a strong consensus (S.D.=.15) that it is of average chronemic agency (.47). A thematic analysis of the interview transcripts led to the identification of several themes that shed light on the way digital media are used by undergraduate students who cope with hundreds of messages and notifications that accumulate every day in their email inboxes, social media accounts, texting apps, and other forms of digital media. Below is a discussion of these themes.

\subsection{The role of chronemic agency in the social norms of mediated communication}

The thematic analysis reveals the role of chronemic agency in the social norms of mediated communication: We find an implicit understanding about high chronemic agency media within the studied social group of undergraduate students in one campus, though this sometimes needs to be explained to people who are not members of this group (e.g. Saral's mentees). We also see evidence that chronemic agency is dynamic. For example, although there is a consensus that texting and phoning are the media of choice for urgent messages, we see extensive diversity in the chronemic agency assigned to other media by different participants in the study. For example, Facetime was assigned maximum (1) chronemic agency by two participants, minimal (0) by three, and .3 and .6 by another two participants. Another example of the dynamic nature of chronemic agency is ReginaFlangee1's description of the decrease in chronemic agency of email during summer break.

\subsection{The responsiveness imperative}

Responsiveness in general, and in mediated conversations, is associated with positive social outcomes such as a sense of belonging, social support and increased social capital [12, 13], while unresponsiveness or slow responsiveness can have the opposite effect [14]. Nevertheless, staying responsive and signaling responsiveness in the "always on" environment where messaging is constant and extensive, requires users to effectively manage the attentional demands that these message place on them, or to pay a personal price such as decreased well-being [15]. Our findings demonstrate how differential chronemic agency enables the participants in our study 
to "budget" their attention effectively while still remaining responsive to their peers and to members of other social circles. We see that each participant develops his or her own strategy of handling the different media and the messages conveyed by them. The strategies of different participants are similar in their handling of high chronemic agency media: these are monitored frequently, and incoming messages and calls are evaluated for urgency. The attention to lower chronemic agency media is allocated in a more individual manner that depends on each person's personal preferences and circumstances, on the time of day, day of the week and time of the year, on how busy or engaged they are, on other social circles that they belong to such as family, work, or religious community, and on idiosyncratic rules such as checking the medium at specific times of the day or at a specific frequency.

Each of the study's participants had a unique strategy for monitoring the different media, but the way messages were handled was quite uniform. This principle was, as described my Michelle1, JohnSmith8, Flower3 and others, minimizing the time between opening the message and responding to it (by answering, or by taking another action). We also learn that there are two main motivations for this minimization of response times. The first reason is process-oriented: A quick response decreases the likelihood of forgetting to respond. This is not surprising in light of the large number of messages and conversational threads processed on a daily basis. Under these circumstances, it is very easy to imagine that the likelihood of forgetting about a message increases with time. It is also in line with previous findings that users have a strong preference for responding quickly to messages [16]. The second reason is the social expectation that messages receive a quick response $[12,13]$. The power of this expectation is underlined by the extensive amount of attention participants such as Jersey8, Barbara7 and Sara1 pay to the "read receipts" that inform the sender that the recipient viewed the message. They report the strong pressure to respond as quickly as possible and thus to prevent a delay between the point in time when the sender knows that the recipient saw the message, and the time the sender receives a response. If the delay is long, this might be interpreted negatively. Macarena5's elaborate process of reading a Snapchat message "under the radar" of the application's servers is an extreme example of the importance of this issue.

\subsection{The shifting nature of (a)synchronicity}

The finding that participants in the study each assign a unique set of chronemic agencies to the media they use marks a subtle but important change in the way personal communication media are used. Until the emergence of mobile media such as the mobile phone and later the smartphones, most communication media could be classified as either synchronous (e.g. phoning) or asynchronous (e.g. email), and most media were located at a specific physical location (e.g home, workplace). Synchronous media such as the telephone were attended to if the person was in the vicinity of the medium, or the message was shifted to an asynchronous form (e.g. message on an "answering machine" or a note left by a person who took the call). Asynchronous media were attended to at specific times. Work email and the fax were checked when employees were in the office, private email was attended to from home. Letters and memos were similarly attended to.

The reality described by the participants of this study is different. All media are, practically, synchronous, since the messages are transmitted almost instantaneously, and can be accessed on a mobile device that is carried on their person at all times. The decision as to when to access the messages is no longer determined by location or by medium, and is now a decision that users take themselves, based on a complex, chronemic agency-based system described here. In the not too distant past the only key synchronous communication media were face-to-face conversations, and telephone calls. In the reality described by our study's participants, these two media are no longer that unique. It is tempting to label this the "death of asynchronicity", though it is just as apt to describe this as the "death of synchronicity", since almost all messages can be saved for a later time, and the pace of mediated conversations can be modulated by the participants.

Our findings suggest that classifying media as synchronous or asynchronous is more fluid than assumed in the literature. For example, a quick search of the leading Journal of Computer-Mediated Communication identified dozens of papers published since 2010 that use the terms synchronous and asynchronous to classify media. Is this classification, so commonly used in the literature, still valid? Furthermore, these findings inform the research thread of affordances in communication media [17], and provide further evidence to the fluidity of affordances.

\subsection{Dealing with communication overload}

Communication overload has been described [18] as comprising of seven conceptual dimensions: Compromising message quality, having many distractions, using many ICTs, feeling responsible to respond, pressuring for decisions, overwhelming with 
information, and piling up of messages. Our findings shed light on several of these dimensions. In particular, we see extensive evidence for the "feeling responsible to respond" component, for the use of many ICT's, and for having many distractions. Accordingly, we see that differential chronemic agency provides a system that moderates the negative effect of many aspects of communication overload by prioritizing the different media, by responding to less time-sensitive messages at specific, more convenient, times, and by avoiding excess distractions.

\subsection{Conclusion}

This exploratory study set out to better understand whether one of the ways through which users manage the constant influx of large numbers of messages on many different media is by prioritizing messages that arrive on only a small number of the many communication media they use. This prioritization is based on a general understanding that these media are assigned high chronemic agency, and that they are where urgent messages will be sent to. We proposed the term chronemic agency of a medium to describe the relative power that messages and notifications that are received through the medium have on the recipient's attention and reaction time. The goals of this study were to look for evidence for the assignment of chronemic agency by users, and then to better understand chronemic agency and its implications for our understanding of everyday communication norms, and for communication theory. We found clear evidence that our participants assign differential chronemic agency to different media, that there is a consensus to assign high chronemic agency to a small number of media (telephoning and texting), and that chronemic agency of other media is lower and less uniform. Our findings set the foundation for using chronemic agency as a tool to better understand mediated conversation. Chronemic agency has a role in the social norms of mediated communication by overloaded users who deal with the blurring boundaries between synchronous and asynchronous media.

\section{Acknowledgements}

We wish to thank Matthew McGlone and Maxim Baryshevtsev for helpful discussions. This study received funding from the Open University Research Authority.

\section{References}

[1] A. Lenhart, "Teens, social media \& technology overview 2015," Pew Research Center, Apr. 2015.

[2] S. Greenwood, et al., "Social media update 2016," Pew Research Center, Nov. 2016.

[3] A. Smith, "U.S. smartphone users in 2015," Pew

Research Center, Apr. 2015.

[4] Y. M. Kalman and G. Ravid, "Filing, piling, and everything in between: The dynamics of E-mail inbox management," J. Assoc. Inf. Sci. Technol., vol. 66, no. 12, pp. 2540-2552, 2015.

[5] A. Smith, "Americans and text messaging," Pew Research Center, Sep. 2011.

[6] Deloitte US, "2016 Global mobile consumer survey: US edition," Deloitte US, 2016.

[7] T. J. Bruneau, "Chronemics and the verbalnonverbal interface," in The Relationship of Verbal and Nonverbal Communication, Walter de Gruyter, 1980, pp. 101-117.

[8] F. Cooren, "The organizational world as a plenum of agencies," in Communication as organizing: Empirical and theoretical explorations in the dynamic of text and conversation, F. Cooren, et al., Ed., New York: Routledge, 2013, pp. 81-100.

[9] M. G. Flaherty, The textures of time: Agency and temporal experience. Temple University Press, 2011.

[10] A. Bagnoli, "Beyond the standard interview: the use of graphic elicitation and arts-based methods," Qual.

Res., vol. 9, no. 5, pp. 547-570, Nov. 2009.

[11] V. Braun and V. Clarke, "Using thematic analysis in psychology," Qual. Res. Psychol., vol. 3, no. 2, pp. 77$101,2006$.

[12] N. B. Ellison, et al., "Cultivating social resources on social network sites: Facebook relationship maintenance behaviors and their role in social capital processes," J. Comput.-Mediat. Commun., vol. 19, no. 4, pp. 855-870, Jul. 2014.

[13] M. Seo, et al., "Frequent interaction and fast feedback predict perceived social support: Using crawled and self-reported data of facebook users," J. Comput.-Mediat. Commun., vol. 21, no. 4, pp. 282-297, Jul. 2016.

[14] Y. M. Kalman and S. Rafaeli, "Online pauses and silence: Chronemic expectancy violations in written computer-mediated communication," Commun. Res., vol. 38, no. 1, pp. 54-69, 2011.

[15] L. Reinecke et al., "Digital stress over the life span: The effects of communication load and internet multitasking on perceived stress and psychological health impairments in a German probability sample," Media Psychol., vol. 20, no. 1, pp. 90-115, Jan. 2017.

[16] Y. M. Kalman and S. Rafaeli, "Email chronemics: unobtrusive profiling of response times," in 38th Hawaii International Conference on System Sciences, Big Island, Hawaii, 2005.

[17] J. W. Treem and P. M. Leonardi, "Social media use in organizations: Exploring the affordances of visibility, editability, persistence, and association," Ann. Int. Commun. Assoc., vol. 36, no. 1, pp. 143-189, Jan. 2013.

[18] K. K. Stephens, et al., "Reconceptualizing communication overload and building a theoretical foundation," Commun. Theory, to be published. 